\title{
A Study of Hematological Malignancies in Tripura Medical College \& Dr. BRAM Teaching Hospital
}

\author{
Nabaneet Majumder' ${ }^{1}$ Sayandeep Nath ${ }^{2}$ \\ ${ }^{1,2}$ Department of Pathology, Tripura Medical College \& Dr BRAM Teaching Hospital, Tripura, Agartala, India.
}

\section{ABSTRACT}

\section{BACKGROUND}

Hematological malignancies are a major health problem in our society due to their high mortality and morbidity. It includes diverse and biologically distinct subgroups and can be defined as clonal neoplasm of hematopoietic stem cells characterized by uncontrolled proliferation of immature cells in bone marrow and spilling into peripheral blood. The patterns of subtypes of hematological malignancies vary in different populations and geographic areas because of genetic, environmental, dietary and social factors. Research of epidemiological features of leukemia are poorly reported in Tripura. The objective of the study was to get an idea about the pattern of hematological malignancies in our institute which will help to get an idea of the disease in our state and find out the incidence of hematological malignancies in Tripura Medical College, a tertiary care hospital in Tripura.

\section{METHODS}

This is a one-year prospective study, conducted in the Department of Pathology, Tripura Medical College \& DR BRAM teaching hospital from July 2017 to June 2018. Medical records of all patients admitted to our hospital and diagnosed as a case of hematological malignancy were reviewed for demographic and clinical characteristics at diagnosis. Data analysis was performed by using statistical package for social sciences (SPSS) version 16.

\section{RESULTS}

Our study included 19 diagnosed cases of hematological malignancies, of which 15 were of acute leukemia and 3 cases were of chronic leukemia. Amongst the cases of acute leukemia, 9 were diagnosed as acute myeloid leukemia (AML) whereas 6 were diagnosed as acute lymphoblastic leukemia (ALL). Out of chronic leukemic cases, all were diagnosed as CML. We reported a solitary case of multiple myeloma in a elderly male patient. Present study showed male predominance, invariably affecting the adults. Commonest form of leukemia in adults were AML followed by chronic myeloid leukemia (CML) whereas all cases of ALL were found in children.The most common peripheral blood finding in hematological malignancy was pancytopenia followed by bicytopenia.

\section{CONCLUSIONS}

In our study, acute leukemia was the most common subtype of leukemia in all age groups. Of all leukemia cases, majority were of AML. Demographic characteristics were almost the same as other regional reports. Our observation is mostly comparable with other studies from India and the neighborhood countries. The differences observed in few demographic parameters may be due to geographic variation and genetic difference of study population.

\section{KEY WORDS}

Hematological Malignancies, Acute Leukemia, Chronic Myeloid Leukemia
Corresponding Author:

Dr. Sayandeep Nath,

Assistant Professor,

Department of Pathology,

Tmc \& Dr BRAM Teaching hospital,

Tripura, Agartala, India.

E-mail: sayandpn@gmail.com

DOI: $10.14260 / j e m d s / 2021 / 502$

How to Cite This Article:

Majumder N, Nath S. A study of hematological malignancies in Tripura medical college \& Dr. BRAM teaching hospital. J Evolution Med Dent Sci 2021;10(31):2456-2459, 10.14260/jemds/2021/502

Submission 05-04-2021, Peer Review 07-07-2021, Acceptance 13-07-2021, Published 02-08-2021.

Copyright (c) 2021 Nabaneet Majumder et al. This is an open access article distributed under Creative Commons Attribution License [Attribution 4.0 International (CC BY 4.0)] 


\section{BACKGROUND}

Developing countries bear more than half of global cancer because $70 \%$ of world's population live in these countries. Worldwide, leukemias constitute $3 \%$ of all malignancies whereas in India, it constitutes $3.5 \%$ of all malignancies. ${ }^{1,2}$ Hematologic malignancies represent a heterogeneous group of pathologies that share the common involvement of bone marrow cells or the lymphatic system. ${ }^{3}$ Leukemias are heterogeneous group of hematopoietic malignancies that include diverse and biologically distinct subgroups. ${ }^{4}$ Acute leukemias afflict both adults and children while chronic leukemias afflicts adults mainly.

Acute myeloid leukemia is the most common acute leukemia in adults, accounting for $80 \%$ of cases in this group. The incidence of AML increases with age, from 1.3 per 100000 population in patients less than 65 years old to 12.2 cases per 100000 population in those over 65 years. Although advances in the treatment of AML have led to significant improvements in outcomes for younger patients, prognosis in the elderly who account for the majority of new cases remains poor. Even with current treatments, as much as $70 \%$ of patients 65 years or older will die of this disease within 1 year of diagnosis. ${ }^{5} \mathrm{AML}$ can arise in patients with an underlying hematological disorder, or as a consequence of prior therapy (for example, exposure to topoisomerases II, alkylating agents or radiation). However, in majority of cases, it appears as a de novo malignancy in previously healthy individuals. ${ }^{5}$

Acute lymphoblastic leukemia is a malignant transformation and proliferation of lymphoid progenitor cells in the bone marrow, blood and extramedullary sites. While 80 $\%$ of ALL occurs in children, it represents a devastating disease when it occurs in adults. The pathogenesis of ALL involves the abnormal proliferation and differentiation of a clonal population of lymphoid cells. Studies in the pediatric population have identified genetic syndromes that predispose to a minority of cases of ALL, such as Down syndrome, Fanconi anemia, bloom syndrome, ataxia telangiectasia and nijmegen breakdown syndrome. Other predisposing factors include exposure to ionizing radiation, pesticides, certain solvents or viruses such as Epstein-Barr virus and human immunodeficiency virus (HIV). However, in majority of cases, it appears as a de novo malignancy in previously healthy individuals. Chromosomal aberrations are the hallmark of ALL, but are not sufficient to generate leukemia. ${ }^{6}$

CML was the first leukemia discovered, being described around 1840 by David Craigie, John Hughes Bennet, and Rudolph Virchow through autopsies on individuals who had presented with very similar findings, such as hepatosplenomegaly, fever, leukocytosis, and an unusual blood appearance and consistency, described by Alfred Velpeau in the $19^{\text {th }}$ century as "thick blood". ${ }^{7}$ Chronic myeloid leukemia is a myeloproliferative disorder characterized by the neoplastic transformation of the hematopoietic stem cell and the pathognomonic presence of the philadelphia $(\mathrm{Ph})$ chromosome arising from a reciprocal translocation between chromosomes 9 and 22. This balanced chromosomal alteration causes the fusion of the abelson oncogene (ABL) from chromosome $9 q 34$ with the breakpoint cluster region (BCR) on chromosome 22q11.2, $\mathrm{t}(9 ; 22)$ ( $\mathrm{q} 34 ; \mathrm{q} 11.2)$ and induces the formation of a distinct chimeric $B C R$ - $A B L 1$ fusion gene, which in turn translates into a $\mathrm{Bcr}-\mathrm{Abl}$ oncoprotein. CML usually presents in chronic phase (CP), characterized by the clonal expansion of mature myeloid cells. Indeed, all untreated patients will eventually progress to a lethal blast phase (BP) that is sometimes preceded by an accelerated phase (AP). ${ }^{8}$

Multiple myeloma (MM) is a disorder caused by neoplastic proliferation of a clone of malignant plasma cells. The characteristic features include accumulation of monoclonal plasma cells in the bone marrow associated with monoclonal immunoglobulin (Ig) synthesis and osteolytic bone lesions. It comprises $13 \%$ of hematologic malignancies. MM is a disease of the elderly, with the peak age of 60 - 70 years at presentation. In spite of advances in treatment, MM remains to be a therapeutic challenge. Though most patients of MM are symptomatic at presentation needing prompt treatment, about 10 - $15 \%$ may remain asymptomatic in whom treatment may be delayed. ${ }^{9}$

The patterns of subtypes of leukemia vary in different populations and geographic area. The spectrum of hematological malignancy is dependent upon various genetic, environmental, dietary and social factors. In developed countries, the existence of cancer registries makes it possible to know the number of cases of hematological malignancies whereas in developing countries where there is no cancer registry most often, epidemiological data are provided by descriptive studies in care and / or diagnostic services. ${ }^{3}$ Accurate reporting of the cancer profile from a region has several limitations especially in a developing country. This is because of improper record keeping, lack of stable population as well as lack of basic health care. There are no well documented reports about the epidemiological features of leukemia in Tripura, a north eastern hilly state which has a mixed population of tribes and non tribes with different genetic and cultural background. Here, an attempt has been made to get an idea about the pattern of hematological malignancies on an institution - based study.

\section{Objective}

To find out the incidence of hematological malignancies in Tripura Medical College and BRAM Teaching Hospital, a tertiary health care hospital in Tripura.

\section{METHODS}

It was a one year prospective study, conducted in the Department of Pathology, Tripura Medical College from July 2017 to June 2018. A total of 19 cases of hematological malignancies were diagnosed and included in our study. All the cases were diagnosed on the basis of peripheral blood smear and bone marrow studies wherever it was required. Bone marrow study was taken up for only one case. Smear prepared and stained with Leishman stain and Giemsa stain. One bone marrow was done, which was properly fixed and stained with Leishman stain. The data obtained were analyzed to evaluate the incidence of hematological malignancies in our institution and was compared with the observation of other workers.

\section{Statistical Analysis}

It was performed by using SPSS version 16. 


\section{RESULTS}

Among a total of 19 cases of malignancy, 15 were of acute leukemia, of which 9 were diagnosed as acute myeloid leukemia and 6 were diagnosed as acute lymphoblastic leukemia. Amongst 3 cases of chronic leukemia, all were diagnosed as chronic myeloid leukemia. We reported a case of multiple myeloma in a 70 year old male patient. Males were affected more commonly than females. Overall, adults were more affected than children and the commonest leukemia in adults was AML followed by CML whereas in the children's subgroup, the commonest type was ALL.

\begin{tabular}{|c|c|c|c|c|c|c|c|}
\hline Entities & $\mathbf{n}$ & (\%) & & Male & Female & Adult & $\begin{array}{c}\text { Children }(<15 \\
\text { years) }\end{array}$ \\
\hline AML & 09 & 48 & & 05 & 04 & 09 & Nil \\
\hline ALL & 06 & 32 & & 04 & 02 & Nil & 06 \\
\hline CML & 03 & 15 & & 02 & 01 & 03 & Nil \\
\hline $\begin{array}{l}\text { Multiple } \\
\text { myeloma }\end{array}$ & 01 & 05 & & 01 & Nil & 01 & Nil \\
\hline \multicolumn{8}{|c|}{ Table 1. Distribution of Malignant Cases Reported on Bone Marrow } \\
\hline CML Subtypes & $\mathbf{n}$ & $(\%)$ & Male & Female & Adult & \multicolumn{2}{|c|}{ Children $(<15$ years) } \\
\hline Chronic phase & 2 & 67 & 01 & 01 & 02 & & - \\
\hline $\begin{array}{l}\text { Accelerated } \\
\text { phase }\end{array}$ & 1 & 33 & 01 & 00 & 01 & & - \\
\hline Total & 03 & 100 & 02 & 01 & 03 & & - \\
\hline
\end{tabular}
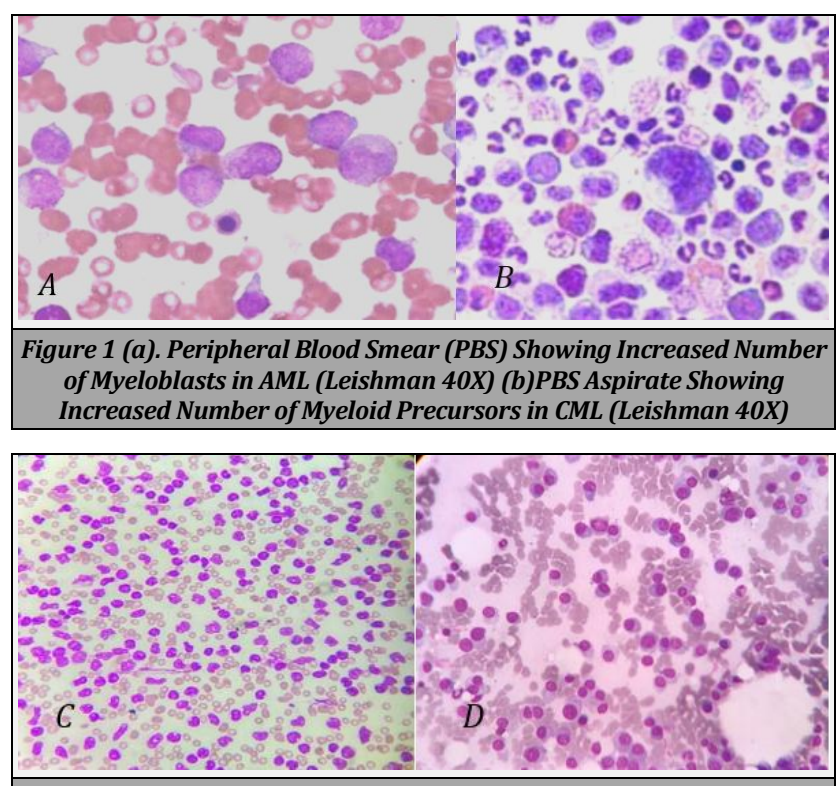

Figure 2(C). PBS Showing Increased Number of Lymphoblasts in ALL (Giemsa 10X), (D) Bone Marrow Showing Plasma Cells \& Plasmablast in Multiple Myeloma (Leishman 10X)

Table 1 shows the number, frequency, gender distribution and age distribution of all cases. The age of patients with AML ranged from 24 years - 75 years with a mean age of 41 years. We reported a total of 9 cases of AML all affecting the adults. Table 2 shows the distribution of 3 cases of CML. 2 cases were in chronic phase, all with blast phase $<5 \%$, 1 case was in accelerated phase with $10-19 \%$ of myeloblasts. We did not find any case of CML in blast crisis (blast phase). The most common peripheral blood finding in hematological malignancy cases were pancytopenia (48 \%) followed by bicytopenia (13\%). Among clinical presentation, splenomegaly ( $52 \%$ ) followed by hepatomegaly (28\%) were common. The most common malignancy was AML (50\%) followed by ALL (34 \%).
On analysis of clinical features of acute leukemia patients, we found pyrexia to be the commonest clinical symptom followed by weakness, pallor, ecchymosis and bone pain. Clinical presentation for chronic leukemias included fever, organomegaly and lymphadenopathy.

\section{DISCUSSION}

Research on epidemiological features of hematological malignancies are poorly reported in Tripura. Our study demonstrates that acute leukemia is commoner than chronic leukemia and the most common morphological type is AML. This observation is consistant with other studies like G.C. et al. ${ }^{10}$ Modak H et al. ${ }^{11}$ and Kulshretra R et al. ${ }^{12}$ We also observed that AML was the most common subtype of acute leukemia which is comparable with the studies by Modak etal and Chen et al. ${ }^{13}$ whereas Kulshretra reported maximum cases of CML. AML is a disease of older age with median age of 60 - 65 years whereas the mean age in our study was 41 years. Different studies in India quoted the median age of AML to be of 39 years.

AML patients showed wide variation in haemoglobin $(\mathrm{Hb}$ $\%$ ), total leukocyte count (TLC) and platelet count. 7 patients had anemia ( $\mathrm{Hb}, 10 \mathrm{gm} / \mathrm{dl})$ and all patients had low platelet count. Interestingly, 02 patients had low to normal TLC with presence of peripheral blasts.

Acute Lymphoid Leukemia: ALL comprises $30 \%$ of childhood cancers worldwide and $75 \%$ cases occur below 10 years and $50 \%$ cases below 5 years. In our study, we reported 6 cases of ALL comprising $40 \%$ cases of acute leukemia and $34 \%$ cases of all leukemia cases. In India, the rate ranges from 9 - $39 \%$ in different series with a higher incidence in Southern India. ${ }^{14}$ ALL is the most common hematological malignancy in pediatric population. ${ }^{15}$ In our study, 5 cases $(83 \%)$ were below 10 years and 1 case was below 15 years. Among ALL, 5 cases had anemia $(\mathrm{Hb}<10 \mathrm{gm} / \mathrm{dl})$ and all had low platelets. 05 cases had TLC $>11000 / \mathrm{mm}^{3}$ and one case had TLC $<4000 / \mathrm{mm}^{3}$.

Chronic Myeloid Leukemia: Worldwide, CML accounts for $20 \%$ of all cases of leukemia. We reported 3 cases of CML comprising $16 \%$ of all leukemias out of which 2 were in chronic phase and 1 in accelerated phase. Different studies of India show frequency of CML ranging from $30-56 \%$ being the commonest form of malignancy in adults. ${ }^{16}$ In our study, all patients had high white blood cell (WBC) count and majority had anemia with high platelets.

Multiple Myeloma: Multiple myeloma is a hematological malignancy usually presenting in the elderly, with a median age of 55 - 60 years. Ghartimagar D et al. ${ }^{17}$ found multiple myeloma represents $15 \%$ of all hematological malignancies whereas it comprised of $5 \%$ of cases in our study. There is no such sex predilection. Males and females are known to be affected equally in other literature though in the current study, one case was reported in a 70 year old male patient. ${ }^{18}$

\section{CONCLUSIONS}

In our study, acute leukemia was the most common subtype of leukemia in all age groups. Of all leukemia cases, majority were of AML. In CML, chronic phase was the commonest of all types 
which is very much comparable with other studies and blast crisis (blast phase) to be the unusual finding. Demographic characteristics were almost the same as other regional reports. Our observation is mostly comparable with other studies from India and the neighborhood countries. The differences observed in few demographic parameters may be due to geographic variation, food habit and genetic difference of study population.

Data sharing statement provided by the authors is available with the full text of this article at jemds.com.

Financial or other competing interests: None.

Disclosure forms provided by the authors are available with the full text of this article at jemds.com.

\section{REFERENCES}

[1] WHO. Health situation in South East Asia region 19941997. New Delhi: Regional Office for SEAR 1999.

[2] Ferlay J, Shin HR, Bray F, et al. Estimates of worldwide burden of cancer in 2008: GLOBOCAN 2008. Int J Cancer 2010;127(12):2893-917.

[3] Refeno V, Hasiniatsy NRE, Ramahandrisoa AVN, et al. Epidemiology and clinical aspects of hematological malignancies at the military hospital of Antananarivo. Int J Res Med Sci 2019;7(6):2037-44.

[4] Petridou E, Pourtsidis A, Trichopoulos D, Leukemias. In: Textbook of Cancer Epidemiology. Oxford: Oxford University Press 2008.

[5] De Kouchkovsky I, Abdul-Hay M. Acute myeloid leukemia: a comprehensive review and 2016 update. Blood Cancer J 2016;6(7):e441.

[6] Terwilliger T, Abdul-Hay M. Acute lymphoblastic leukemia: a comprehensive review and 2017 update. Blood Cancer J 2017;7(6):e577.

[7] Sampaio MM, Santos MLC, Marques HS, et al. Chronic myeloid leukemia-from the Philadelphia chromosome to specific target drugs: a literature review. World J Clin Oncol 2021;12(2):69-94.

[8] Bonifacio M, Stagno F, Scaffidi L, et al. Management of chronic myeloid leukemia in advanced phase. Front Oncol 2019;9:1132.

[9] Diwan AG, Gandhi SA, Krishna K, et al. Clinical profile of the spectrum of multiple myeloma in a teaching hospital. Med J DY Patil Univ 2014;7(2):185-8.

[10] D'Costa G, Siddiqui HM, Pradhan RM, et al. Pattern of leukemias: a ten-year incidence study of 242 cases. J Postgrad Med 1989;35(4):191-5.

[11] Modak H, Kulkarni SS, Kodakol GS, et al. Prevalence and risk of leukemia in the multi-ethnic population of North Karnataka. Asian Pac J Cancer Prev 2011;12(3):671-5.

[12] Kulshetra R, Sah SP. Pattern of occurance of Leukemia at a teaching hospital in eastern region of Nepal-a six year study. JNMA J Nepal Med Assoc 2009;48(173):35-40.

[13] Chen BA, Huang ZH, Zhang XP, et al. An epidemiological investigation of leukemia incidence between 2003 and 2007 in Nanjing, China. Hematol Oncol 2010;3:21.

[14] Bhutani M, Vora A, Kumar L, et al. Lympho-hematopoietic malignancies in India. Med Oncol 2002;19(3):141-50.

[15] Prajapati Z, Kokani MJ, Gonsai RN. Clinicoepidemiological profile of hematological malignancies in pediatric age group in Ahmedabad. Asian J Oncol 2017;3:54-8.

[16] Kumar L, Sagar TG, Maitreyan V, et al. Chronic granulocytic leukemia. A study of 160 cases. J Assoc Physicians India 1990;38(12):899-902.

[17] Ghartimagar D, Ghosh A, Narasimhan R, et al. Pattern of hematological and non-hematological malignancies in bone marrow in a tertiary care hospital in Nepal-11 years study. Nepal Med Col J 2012:14(3):187-92.

[18] Fousad C, Gangadharan KV, Abdulla MC, et al. Clinical profile of multiple myeloma in South India. Indian J Med Paediatr Oncol 2018;39:62-6. 